Article

\title{
A Simple and Reliable PDMS and SU-8 Irreversible Bonding Method and Its Application on a Microfluidic-MEA Device for Neuroscience Research
}

\author{
Yufei Ren ${ }^{1, *}+{ }^{\dagger}$, Shun-Ho Huang ${ }^{2, \dagger}$, Sébastien Mosser ${ }^{4}$, Marc Olivier Heuschkel ${ }^{5}$, \\ Arnaud Bertsch ${ }^{1}$, Patrick C. Fraering ${ }^{4}$, Jia-Jin Jason Chen ${ }^{2,3}$ and Philippe Renaud ${ }^{1}$ \\ Received: 27 October 2015; Accepted: 1 December 2015; Published: 7 December 2015 \\ Academic Editor: Andreas Richter \\ 1 Microsystems Laboratory, École Polytechnique Fédérale de Lausanne, EPFL-STI-IMT-LMIS4, Station 17, \\ Lausanne 1015, Switzerland; arnaud.bertsch@epfl.ch (A.B.); philippe.renaud@epfl.ch (P.R.) \\ 2 Department of Biomedical Engineering, National Cheng Kung University, Tainan 70101, Taiwan; \\ shunho.huang@mail.huji.ac.il (S.-H.H.); chenjj@mail.ncku.edu.tw (J.J.C.) \\ 3 Medical Device Innovation Center, National Cheng Kung University, Tainan 70101, Taiwan \\ 4 Brain Mind Institute and School of Life Sciences, École Polytechnique Fédérale de Lausanne, \\ EPFL-SV-BMI-CMSN, Station 15, Lausanne 1015, Switzerland; sebastien.mosser@epfl.ch (S.M.); \\ patrick.fraering@epfl.ch (P.C.F.) \\ 5 Qwane Biosciences SA, Lausanne 1015, Switzerland; marc.heuschkel@qwane.com \\ * Correspondence: yufei.ren@epfl.ch; Tel.: +41-21-693-6727 \\ + These authors contributed equally to this work.
}

\begin{abstract}
Polydimethylsiloxane (PDMS) and SU-8 are currently two very commonly used polymeric materials in the microfluidics field for biological applications. However, there is a pressing need to find a simple, reliable, irreversible bonding method between these two materials for their combined use in innovative integrated microsystems. In this paper, we attempt to investigate the aminosilane-mediated irreversible bonding method for PDMS and SU-8 with X-Ray Photoelectron Spectroscopy (XPS) surface analysis and bonding strength tests. Additionally, the selected bonding method was applied in fabricating a microelectrode array (MEA) device, including microfluidic features, which allows electrophysiological observations on compartmentalized neuronal cultures. As there is a growing trend towards microfluidic devices for neuroscience research, this type of integrated microdevice, which can observe functional alterations on compartmentalized neuronal culture, can potentially be used for neurodegenerative disease research and pharmaceutical development.
\end{abstract}

Keywords: PDMS; SU-8; bonding technology; surface silanization; XPS analysis; tensile strength test; integrated microfluidic-MEA device; compartmentalized neural cell culture; neural activity recording

\section{Introduction}

Polydimethylsiloxane (PDMS) is an elastomeric silicone material that is widely used for rapid prototyping microfluidic systems and cell-chip devices, due to its chemical inertia, thermal stability, permeability to many gases, simple preparation, optical transparency, and low cost [1]. It can be easily integrated with electrodes, heaters, and sensors, which are fabricated on substrates, to generate multifunctional devices for biomedical applications [2]. SU-8 is an epoxy-based negative photoresist initially developed for the microelectronics industry [3]. Currently, SU-8 becomes widespread in the development of microfluidic devices due to its ease of use, the high aspect ratio it allows to create, its high chemical stability, and mechanical properties [4]. It has become a material of 
choice for microelectromechanical systems (MEMS) and microfluidics, from the fabrication of single components to complete lab-on-chip devices [5]. Since PDMS and SU-8 are both popular materials in the microfabrication field [2,3], researchers have started to integrate PDMS and SU-8 together more and more to benefit from both of their advantages [6-8]. In the fabrication of microfluidic devices made of PDMS, the PDMS component is generally bonded to a glass coverslip to obtain closed microfluidic channels by using an oxygen $\left(\mathrm{O}_{2}\right)$ plasma surface treatment on the PDMS and glass surfaces. This results in a permanent covalent siloxane ( $\mathrm{Si}-\mathrm{O}-\mathrm{Si}$ ) bond between the PDMS and glass surfaces. However, using $\mathrm{O}_{2}$ plasma is not sufficient to bond PDMS and SU-8 surfaces irreversibly as the oxidation of the SU-8 surface does not result in the creation of -SiOH groups.

Recently, different bonding methods for PDMS and SU-8 have been reported in the scientific literature, such as nitrogen plasma treatment [9] or spin coating SU-8 on the PDMS surface [6]. Nevertheless, nitrogen plasma is not available in all labs, and spin coating, which requires layer-by-layer processing during device fabrication, does not provide the flexibility of bonding two fabricated individual devices. Some researchers also choose to bond PDMS and SU-8 reversibly [10]. However, for long-term cell culture devices where liquid leakage is to be avoided during the whole duration of the experimentation, a solid sealing is necessary which reversible bonding cannot provide. Instead of aligning the devices every time before each use, in the case of reversible bonding, irreversible bonding can also save time and effort on repeating micro-scale design alignment for the devices before each use as this step is time-consuming. As it is increasingly demanded of integration between PDMS and SU-8 devices, irreversible bonding of PDMS and SU-8 becomes an important technology.

Silanization is one of the widely-applied surface modification methods. It generates a self-assembled monolayer of alkoxysilane molecule, which has methoxy $\left(\mathrm{CH}_{3} \mathrm{O}-\right)$, or ethoxy $\left(\mathrm{CH}_{3} \mathrm{CH}_{2} \mathrm{O}-\right)$ groups onto a substrate. Aminosilane, one of the alkoxysilane molecules, has an amino $\left(\mathrm{NH}_{2}-\right)$ group as well. Aminosilane-mediated silanization has been applied on silica surfaces as a coupling agent for functionalization due to its bifunctional nature [11]. (3-Aminopropyl)triethoxysilane (APTES) is one of the highly-selective effective aminosilanes, which has been widely applied in bonding materials for microdevice fabrication and protein immobilization for biological applications [12-14]. It has been used for bonding PDMS to various thermoplastic materials including polycarbonate (PC), cyclic olefin copolymer (COC), polymethylmethacrylate (PMMA), polystyrene (PS) and others $[12,15,16]$. SU-8 is an epoxy-based negative photoresist and the epoxy groups that remain on the SU-8 surface could be sufficient to react with the aminosilane molecule from the PDMS surface and form a covalent bond. In this way, PDMS and SU-8 bonding can be realized by introducing APTES molecules between the two materials (Figure 1).

In this paper, we try to find a simple and reliable bonding method for PDMS and SU-8, based on the elements and chemical bonds from the modified PDMS surface through the XPS analysis and the tensile strength test for the SU-8 processed with and without a hard bake step. In addition, we detail this bonding method with a practical microfluidic device example. This device combines a compartmentalized microfluidic device made of PDMS and a microelectrode array (MEA) neural activity recording device with SU-8 as its insulation layer. We name it a microfluidic-MEA device. The design of the electrode array is adapted to the compartmentalized design from the microfluidic PDMS device. This allows us to distinguish the neural activity from different cell populations, which can potentially present different disease status. Axons can be isolated from the neuronal network and be recorded separately. This combination of compartmentalized PDMS device and MEA device provides a multifunctional platform for neuroscience research. Our bonding technology is used to bond the PDMS and SU-8 surfaces irreversibly for this microfluidic-MEA device. The cell compatibility of this bonding method is proved through neural electrophysiological results using this integrated device. It is promising to apply this simple and reliable irreversible bonding method to integrate microdevices with PDMS and SU-8 surfaces for biological use. This type of microfluidic-MEA device has proved an innovative tool for neural electrophysiological studies. 

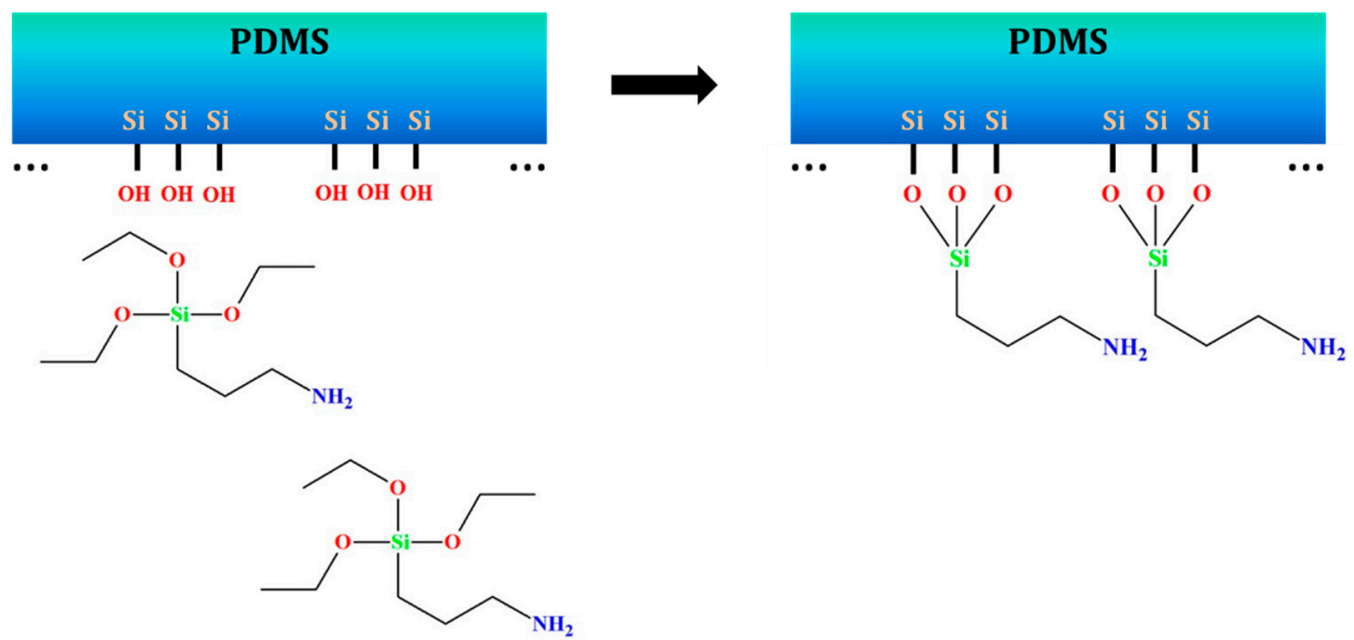

(a)
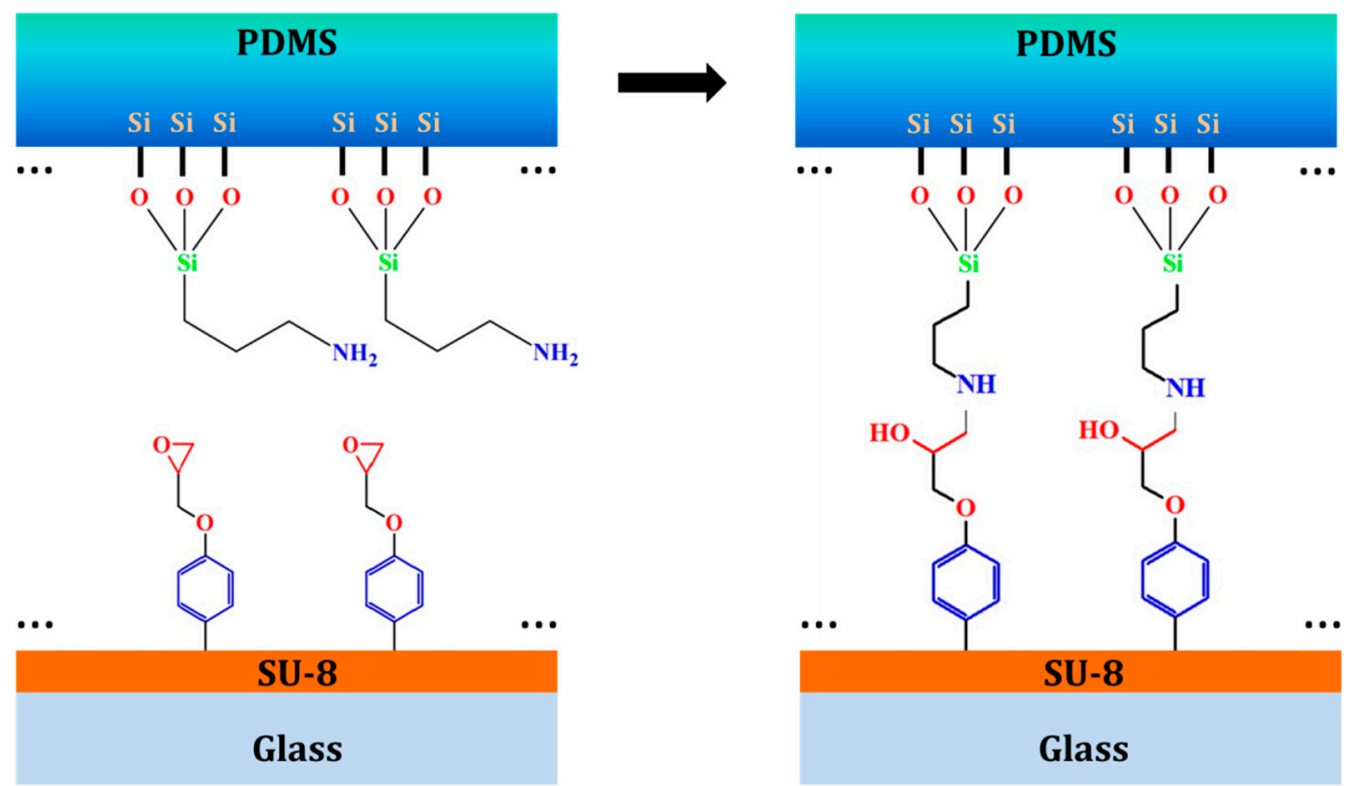

(b)

Figure 1. Theoretical reactions during the bonding. (a) Reaction between APTES molecule and the $\mathrm{O}_{2}$ plasma activated PDMS surface and $(\mathbf{b})$ reaction between the $-\mathrm{NH}_{2}$ group from the APTES molecule on the PDMS surface and the epoxy group from the SU-8 surface.

\section{Materials and Methods}

\subsection{Experimental Materials}

PDMS Sylgard ${ }^{\circledR} 184$ Silicone Elastomer Kit from Dow Corning Corporation (Midland, MI, USA) was sold as two components, the PDMS base and its curing agent. SU-8 (GM 1060) was ordered from Gersteltec Sàrl (Pully, Switzerland). (3-Aminopropyl)triethoxysilane 99\% (APTES) was purchased from Sigma-Aldrich GmbH (Buchs, Switzerland).

\subsection{Bonding Test Preparation}

In this irreversible bonding method, APTES molecules, which are both $\mathrm{CH}_{3} \mathrm{CH}_{2} \mathrm{O}$ - and $\mathrm{NH}_{2-}$ terminated, are bound both to the PDMS and SU-8 interfaces. The $\mathrm{CH}_{3} \mathrm{CH}_{2} \mathrm{O}$ - group reacts with the $-\mathrm{SiOH}$ group from the $\mathrm{O}_{2}$ plasma activated PDMS. The $\mathrm{NH}_{2}$ - group from the other end of the APTES molecule reacts with the epoxy group on the SU-8 surface. Two factors that play important 
roles during this bonding process were investigated experimentally: (1) the way the silanization is carried out, either in a liquid-phase or vapor-phase method; and (2) the degree of reticulation of SU-8 achieved before performing the PDMS and SU-8 bonding step. These two factors have effects on the efficiency of bringing APTES molecules on the PDMS surface and the amount of epoxy groups on the SU-8 surface after the baking process, respectively. Since the microfluidic device bonded with this method will be used for neural cell culture in the future, and the neurons will grow on the surface of SU-8, we decided to have the surface modification on the PDMS surface instead of the SU-8 surface to have less effect on the cell culture.

We prepared our test samples by a combination of PDMS and SU-8 samples under different processed conditions: for the PDMS surfaces, they were first activated by $\mathrm{O}_{2}$ plasma $(50 \mathrm{~W}$, 0.6 mbar, $30 \mathrm{~s}$ ) to create the $-\mathrm{SiOH}$ group on the PDMS surface. After the surface activation, the APTES molecules were brought in contact with the activated PDMS surface immediately. In the literature, different methods to achieve a surface silanization using APTES have been described, such as liquid-phase silanization with deionized (DI) water followed by heating to a temperature of $85^{\circ} \mathrm{C}$ [17], or by mixing with anhydrous toluene [13] or ethanol [12] and vapor-phase silanization [18]. To avoid the use of toxic chemicals, such as toluene [19], and provide a bonding method that is compatible with the requirements of the cell culture on a chip, we decided to use directly $99 \%$ APTES in liquid-phase or vapor-phase for the silanization step. The protocol we used for liquid-phase silanization is the following: PDMS surface was immersed for 5 min into 99\% APTES immediately after the $\mathrm{O}_{2}$ plasma, then washed in DI water and dried. The protocol for vapor-phase silanization is the following: PDMS samples were exposed for $0.5 \mathrm{~h}$ or $1.0 \mathrm{~h}$ of APTES vapor by placing them into a desiccator containing a few drops of liquid $99 \%$ APTES and reaching a vacuum environment so that APTES evaporates.

To obtain SU-8 surfaces showing different degrees of reticulation, some samples were used in the bonding tests without hard bake, while others were submitted to a $2 \mathrm{~h}$ hard bake at $150{ }^{\circ} \mathrm{C}$ on a hotplate. XPS analysis was performed on the surface of the PDMS obtained by different silanization methods, including vapor-phase and liquid-phase. The bonding strength was evaluated for different degrees of reticulation for the SU-8, including SU-8 with and without the hard bake process.

\subsection{Fabrication of Our Integrated Microfluidic-MEA Device}

In order to demonstrate the simplicity of this bonding method and prove its satisfying performance with a real case for long-term biological use, we describe here the detail of this bonding method with our integrated microfluidic-MEA device. This device combines a microfluidic device made of PDMS and a MEA device with a $5 \mu$ m layer of SU-8 as an insulation layer on its top (Figure 2). The PDMS microfluidic device provides the compartmentalized chambers for neuronal culture and the MEA device provides electrodes at the bottom of the chambers allowing neural activity recording. This type of integrated device is designed for electrophysiological studies from compartmentalized neuronal culture [20]. In the following, we will detail the fabrication of this device.

For the compartmentalized PDMS device fabrication, the PDMS base and the curing agent were mixed at a ratio of 10:1. The mixture was poured onto a silicon wafer with structured patterns, degassed in a desiccator for $20 \mathrm{~min}$ until the air bubbles were gone, and cured in an oven at $80{ }^{\circ} \mathrm{C}$ for $1 \mathrm{~h}$ to solidify the PDMS mixture and replicate the pattern on the surface of the PDMS device. Subsequently, a PDMS puncher was used to perforate the PDMS device to create reservoirs, which connect the microchannels to the macro world for the injection of fluids into the device. The PDMS surface was first cleaned using adhesive tape to remove small particles generated from the hole-punching step. It was then sonicated in an ultrasonic bath for $5 \mathrm{~min}$ and the surface was dried. The PDMS device was placed in the $\mathrm{O}_{2}$ plasma machine to activate the surface $(50 \mathrm{~W}, 0.6 \mathrm{mbar}$ for $30 \mathrm{~s})$. This surface modification step creates $-\mathrm{SiOH}$ groups on the PDMS surface. Afterwards, the PDMS device was immediately immersed into the 99\% APTES solution for $5 \mathrm{~min}$ and then washed 
with DI water and dried. The $\mathrm{CH}_{3} \mathrm{CH}_{2} \mathrm{O}$ - groups from APTES molecule can attach and react with the -SiOH group from the activated PDMS surface and form a covalent Si-O-Si bond [21].

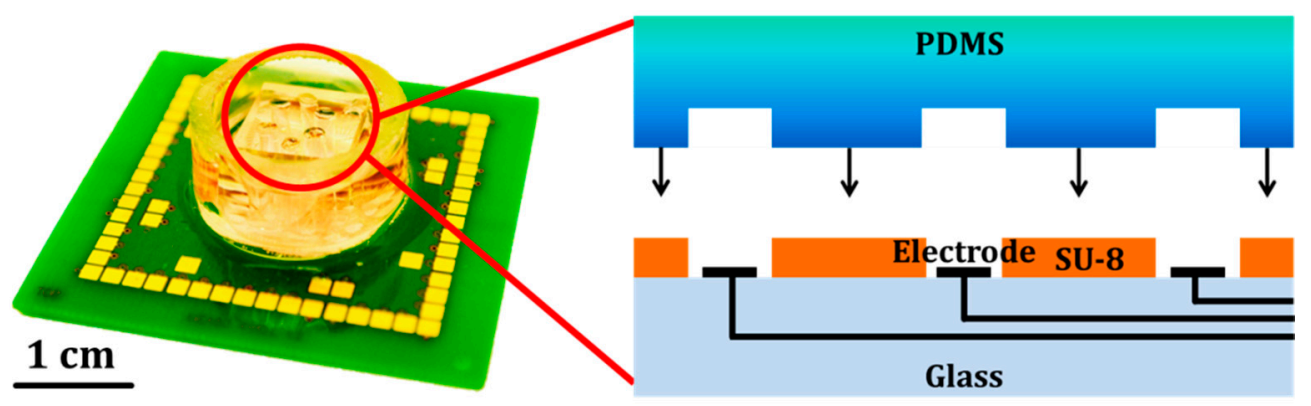

(a)

(b)

Figure 2. The microfluidic-MEA device for neural cell culture. (a) Picture of this integrated microfluidic-MEA device bonded with our PDMS and SU-8 bonding method; and (b) schematic of the cross-section of the device.

For the MEA part in this microfluidic-MEA device, the SU-8 epoxy photoresist was coated on top of the electrode wires, as it acts as an insulation layer between the cell layer and the electrode array layer (Figure 2b). Polymer coatings, such as SU-8, are widely used on metals for insulation and protection for circuit boards and wires in electronic devices to provide high electrical insulation and protection from environmental damage [22]. This SU-8 insulation layer is essential for this neural activity recording device because it can also reduce the parasitic capacitance between the electrode wires and the culture medium (conductive saline solution) for better signal recording [23]. A $5 \mu \mathrm{m}$ thick layer of SU-8 was first spin-coated on the MEA surface, and baked by a hot plate at $60{ }^{\circ} \mathrm{C}$ for $15 \mathrm{~min}$ and another $15 \mathrm{~min}$ at $95^{\circ} \mathrm{C}$ for a soft bake. Then it was exposed to UV light and polymerized by a hot plate at $80^{\circ} \mathrm{C}$ for $20 \mathrm{~min}$ for a post-exposure bake, and developed in PGMEA solvent. The SU-8 surface (without the hard bake process) from the MEA device also needs to be cleaned using isopropyl alcohol to remove the residual particles on the surface. Subsequently, the SU-8 surface was carefully rinsed by DI water and dried.

When the surface modification of the PDMS device has finished, the PDMS device was taken out of the APTES solution, washed by DI water and dried. Then the PDMS device was immediately aligned onto the MEA device under a microscope without touching the silanized surface before alignment was achieved. Afterwards, the microfluidic-MEA device was placed on a flat surface inside an oven and a pressure of $2 \mathrm{~N} / \mathrm{cm}^{2}$ was applied on the top of the device to generate a force between the PDMS and MEA parts to keep these two surfaces fully in contact. The temperature of the oven was slowly increased with a ramp of $2{ }^{\circ} \mathrm{C}$ per min to $150{ }^{\circ} \mathrm{C}$ and kept at $150{ }^{\circ} \mathrm{C}$ for $1 \mathrm{~h}$. Then, the temperature was decreased back to $30^{\circ} \mathrm{C}$ naturally. The reason for increasing and decreasing the temperature slowly is to avoid structural deformations, as well as the generation of cracks inside the SU-8 material, which would create leakage. During this bake time, a chemical reaction occurs between the $-\mathrm{NH}_{2}$ group from the APTES molecule on the PDMS surface and the epoxy group from the SU-8 surface [24]. The bonding for this integrated microfluidic-MEA device was completed.

\subsection{Primary Cortical Neurons Preparation}

Mouse primary cortical neurons were prepared from embryonic day 17 mouse fetal brains. Cortices were digested in a media containing the enzyme papain $(20 \mathrm{U} / \mathrm{mL}$, Sigma-Aldrich $\mathrm{GmbH}$, Buchs, Switzerland) and dissociated by mechanical trituration. Cells were plated in neural cell culture medium: neurobasal medium supplemented with $2 \%$ B27, $1 \%$ L-glutamine, and $1 \%$ penicillin/streptomycin. Cells were plated in polyethyleneimine (PEI, $0.05 \%, v / v$ ) and laminin $(20 \mu \mathrm{g} / \mathrm{mL})$ coated microfluidic devices and were kept at $37^{\circ} \mathrm{C}$ in a humidified $5 \% \mathrm{CO}_{2}$ atmosphere. 
PEI $(50 \%, w / v)$ and laminin were purchased from Sigma-Aldrich GmbH (Buchs, Switzerland). All the materials used for cell culture were purchased from Invitrogen (LuBioScience GmbH, Lucerne, Switzerland).

\section{Results and Discussion}

\subsection{Analysis of Chemical Reactions on the PDMS}

In order to prove the APTES molecules were bound onto the PDMS surface by liquid- and vapor-phase silanization, the elements and the chemical bonds on the PDMS surfaces have been determined by XPS analysis. The samples were analyzed using an ESCA KRATOS AXIS ULTRA Surface Analysis System. Data were analyzed by software MultiPak Version 9.5 (ULVAC-PHI Inc., Chigasaki, Japan). All spectra were calibrated in reference to the aliphatic $C$ 1s component at a binding energy of $285.0 \mathrm{eV}$ [25].

Among all the molecules present in the bonding protocols studies here, the $\mathrm{N} 1 \mathrm{~s}$ is only present in the $-\mathrm{NH}_{2}$ group of the APTES molecule, so its presence in the XPS spectra proves the presence of the APTES molecule on the tested sample. Figure 3 shows the $\mathrm{N} 1 \mathrm{~s}$ binding energy from different samples: Sample 1 corresponds to a PDMS sample without any chemical surface treatment, Sample 2 is a liquid-phase silanized sample under $5 \mathrm{~min}$ immersion in 99\% APTES, Samples 3 and 4 are vapor-phase-silanized PDMS surfaces, exposed for $0.5 \mathrm{~h}$ and $1.0 \mathrm{~h}$ to the APTES vapor, respectively. A peak in the XPS spectra close to an energy of $400 \mathrm{eV}$ corresponding to the $\mathrm{C}-\mathrm{NH}_{2}$ bond can be seen only for Sample 2, proving the efficiency of the liquid-phase silanization method. No significant peak at this value of the energy can be observed for the other samples, which indicates that tested vapor-phase silanization of PDMS is inefficient in our experiment. Interestingly, the composition measurement of the main elements from Sample 2 (C 1s 44.7\%, O 1s 30.9\%, N 1s 7.3\%, Si 2p 17.1\%) is very close to the main elements composition of the APTES molecule (C 1s $48.9 \%$, O 1s $21.7 \%, \mathrm{~N} 1 \mathrm{~s}$ $6.3 \%, \mathrm{Si} 2 \mathrm{p} 12.7 \%$ ). This is a further indication of the presence of the APTES molecules on the surface of this PDMS sample after the liquid-phase silanization.

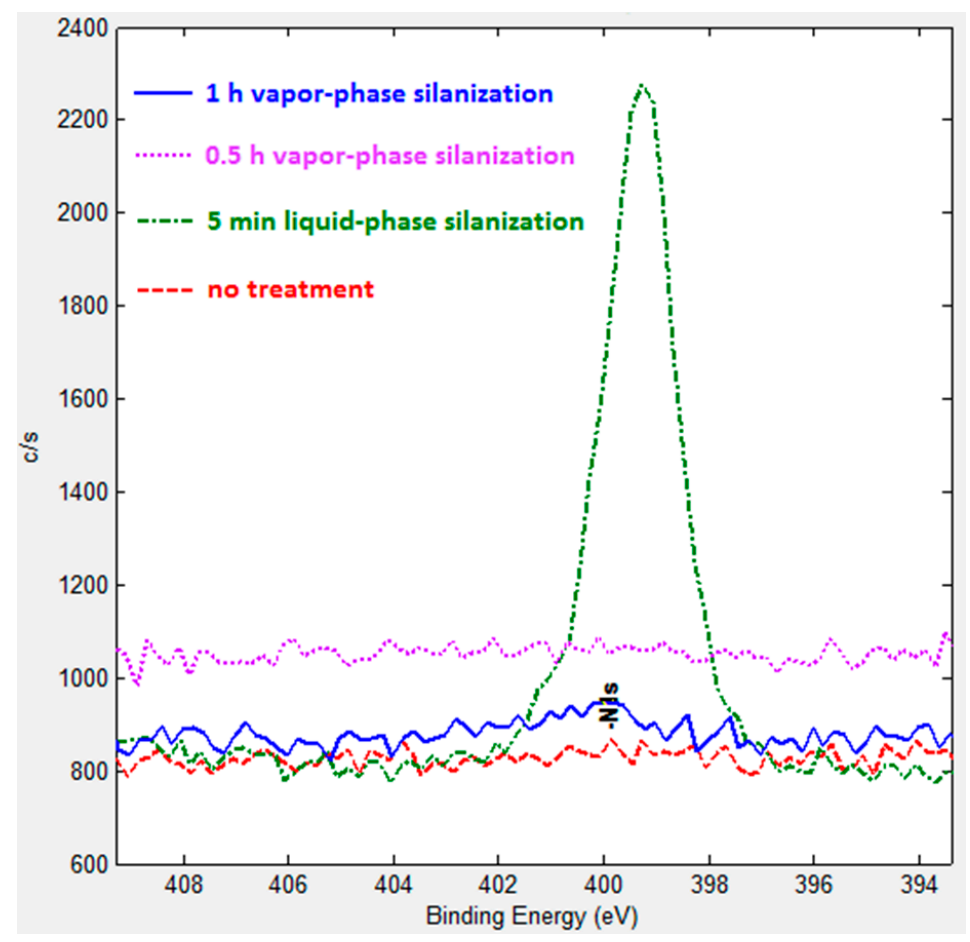

Figure 3. The N 1s spectra of the PDMS surfaces by XPS under different surface modifications. 


\subsection{Manual Tensile Strength Test}

SU-8 without hard bake can provide a larger number of epoxy groups and better contact for adhesion, which can potentially achieve a better bonding quality. To verify the difference of SU-8 with and without hard bake in our bonding method, we performed a manual tensile strength test on the assembled PDMS and SU-8 samples obtained by combining PDMS samples resulting from liquid-silanization of APTES and SU-8 samples with and without hard bake. The samples submitted to the tensile test were prepared by bonding a $1 \mathrm{~cm}$ diameter PDMS device to a $5 \mu \mathrm{m}$ thick layer of SU-8 deposited on glass (Figure 4a,b). The contact surface between PDMS and SU-8 was $0.785 \mathrm{~cm}^{2}$ in circular shape. The surface of the PDMS device was activated by $\mathrm{O}_{2}$ plasma and processed with liquid-phase APTES surface silanization. SU-8 samples with and without hard bake were both individually bonded with the modified PDMS device and tested in this tensile strength test. The testing set-up (Figure 4c) was built manually. As shown in Figure 4c, a scale carrying a piece of metal of $3.530 \mathrm{~kg}$ was attached to a transparent plastic holder with a square hole on its top to maintain the tested the PDMS sample part. A lifting-jack was inserted between the plastic holder and the glass part of the tested sample, moved in the vertical direction and can lift up to $6 \mathrm{~kg}$ (Figure $4 \mathrm{~d}$ ). With this set-up, by lifting up the SU-8-coated glass part of the sample, we created a separating force between the SU-8 and the PDMS that was attached to the metal plate. This force was measured by the change of mass indicated by the scale. With this tensile strength test, we observed the difference in bonding strength between the SU-8 with and without hard bake. The samples combined with hard baked SU-8 broke at the interface of PDMS and SU-8 during the lifting by the lifting-jack. However, the samples combined with SU-8 without hard bake showed a good adhesion until the separation process applied reached $440 \mathrm{kPa}$, without failure, which was the highest value the test set-up can provide. This is substantially higher than the working pressure for typical microfluidic devices. This manual tensile test revealed that SU-8 is required without a hard bake before contacting with the silanized PDMS surface. After the two surfaces were in contact and processed by the final baking step $\left(150^{\circ} \mathrm{C}\right.$ for $\left.1 \mathrm{~h}\right)$, a strong irreversible bonding which can reach $440 \mathrm{kPa}$ was made between the PDMS and SU-8. This manual tensile strength test setup is flexible and convenient for quick tensile testing in the lab as a preliminary result.

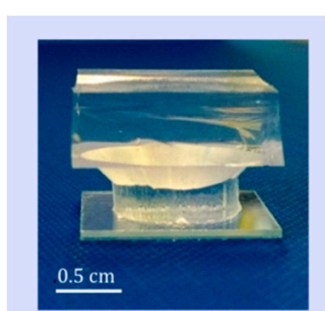

(a)

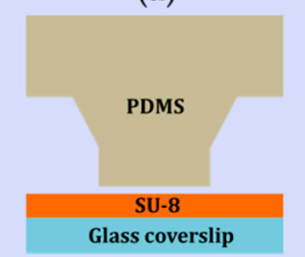

(b)

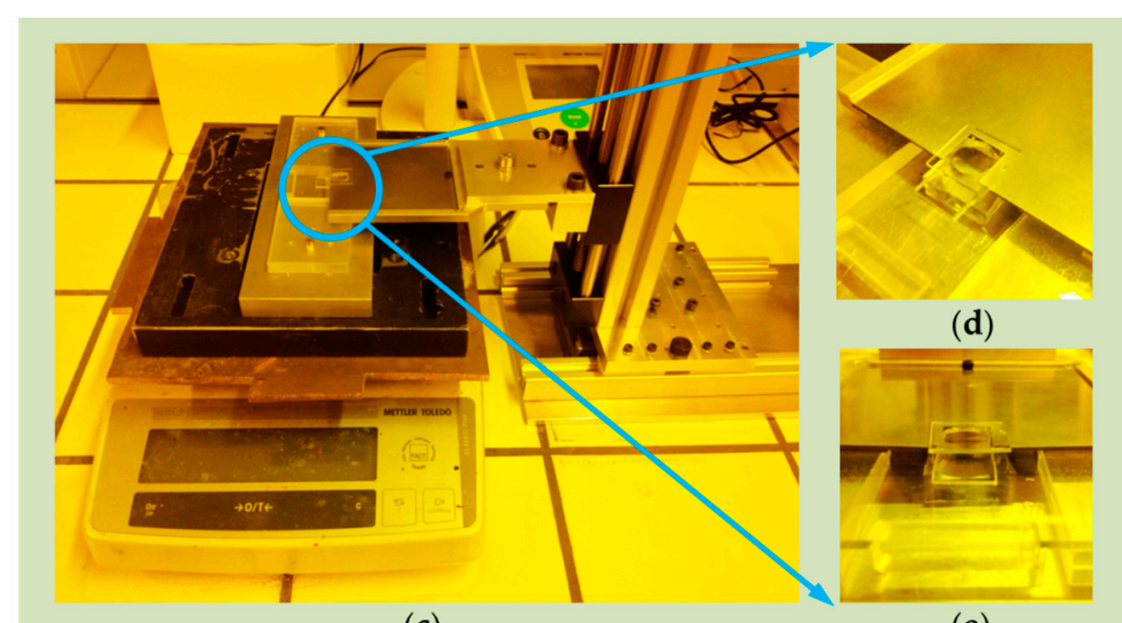

(c) (e)

Figure 4. PDMS and SU-8 bonding testing sample $(\mathbf{a}, \mathbf{b})$, and the setup for the manual tensile strength test (c-e). (a) PDMS and SU-8 samples for the bonding strength test. (b) Schematic of the testing sample. The lower part is a piece of $1 \mathrm{~mm}$ thick glass coated with a $5 \mu \mathrm{m}$ thick SU-8. The upper part is a piece of PDMS obtained by molding. This setup (c) for the manual tensile strength test includes a scale, a piece of metal mass with a plastic PDMS chip fixer on top, and a lifting-arm (c). Detail of the manual tensile test set-up during test (d) and its side view (e). 


\subsection{Ultimate Tensile Strength Test for the Bonding}

To investigate further the limit of the tensile strength for our bonding method by using liquid-phase silanized PDMS surface and the SU-8 surface without hard bake, a tensile test machine from Walter + Bai AG (Löhningen, Switzerland) was used and the test was conducted under force control until the breaking point of the bonding. The tested sample was attached between two metal sample holders (Figure 5). The separating force on the tested sample was increased by the machine until the PDMS and SU-8 bonding interface broke. The tensile strength force measured during the tensile strength test was $116 \pm 5 \mathrm{~N}$, with most of the deformation occurring on the PDMS. This result corresponds to a stress of around $1.5 \mathrm{MPa}$ at the point where the breaking occured. This is reasonable when we consider the tensile strength of PDMS which is much higher than this bonding breaking point [26]. The published result of tensile strength for PDMS and SU-8 bonding reach around $1 \mathrm{MPa}$ [13]. Compared to this value, our method provided a slightly higher tensile strength of the irreversible bonding.

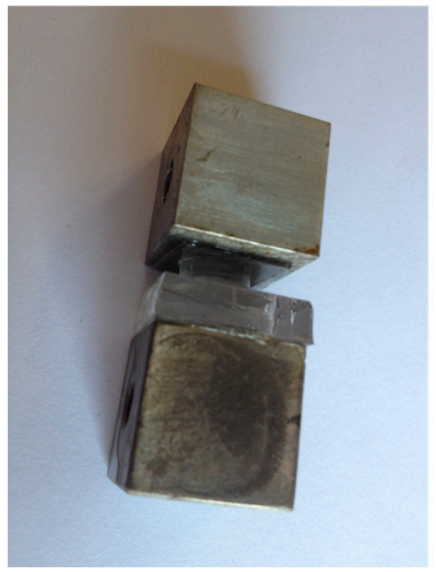

(a)

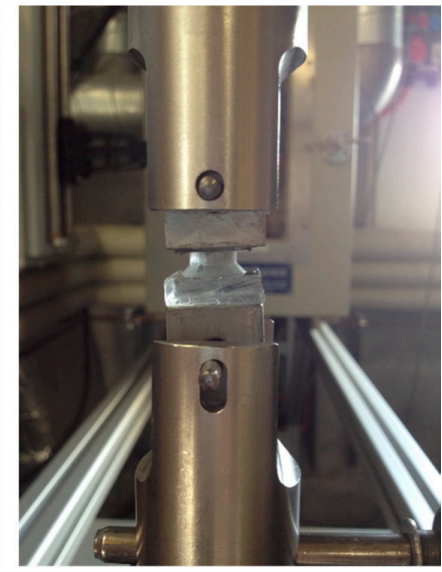

(b)

Figure 5. Tensile strength test for the bonding. (a) Test sample was fixed between the two metal parts from the tensile test machine for the tensile strength test; (b) A sample during the test.

\subsection{PDMS and SU-8 Bonding Method Applied to an Integrated Microfluidic-MEA Device}

The compartmentalized PDMS device has three chambers and each chamber has two columns of 10 electrodes (Figure 6a). Each two chambers are connected by junction channels of $20 \mu \mathrm{m}$ width, $10 \mu \mathrm{m}$ height and $500 \mu \mathrm{m}$ length. The low dimensions on width and height allow the microfluidic PDMS device to segregate the neuronal soma in one chamber and allow its axon to grow through the junction channel to the neighbor chamber. We placed mouse primary cortical neural cells into the two lateral chambers (Chambers 1 and 3) and the axons from the two lateral cell populations grew through the junction channels and built a neuronal network in the middle chamber (Chamber 2). The electrodes from the MEA device were made of platinum ( $50 \mu \mathrm{m}$ diameter), which allow simultaneous recording of extracellular potentials from the neuronal culture. At the same time, both the microfluidic PDMS and MEA devices were designed to provide a good fitting of their functions and their integration (Figure 6b).

The device needed to be sterilized before its use for cell culture. $70 \%$ ethanol was injected into the reservoirs using a syringe, which generated a flow inside the device to both wash away the particles remaining inside the chambers and junction channels and to sterilize them. It was kept inside the device for $20 \mathrm{~min}$, and then sucked out by a vacuum aspirator. The ethanol was then replaced with DI water. The chambers and junction channels were washed with DI water three times and emptied by using the vacuum aspirator each time. The device was then placed in a sterile petri dish $(100 \mathrm{~mm}$ diameter) and exposed under UV light for $1 \mathrm{~h}$. Like normal neural cell culture in well plates, the 
cell adhesion surface inside the device needs to be treated with a coating solution for faster neuronal adhesion and more homogenous cell distribution. The coating solution, $0.05 \%(v / v)$ PEI, was injected into the device from the reservoirs and kept in the chambers and junction channels for $2 \mathrm{~h}$ at $37^{\circ} \mathrm{C}$ inside a sterile, closed petri dish. Then, the PEI coating solution was removed and the device was washed three times with DI water and replaced with a laminin $(20 \mu \mathrm{g} / \mathrm{mL})$ coating solution and kept at $37^{\circ} \mathrm{C}$ for another $20 \mathrm{~min}$. Finally, the laminin coating solution was removed, the device was washed three times with DI water, and replaced with neural culture medium (see recipe in materials and methods section) and kept inside a petri dish in the incubator $\left(37^{\circ} \mathrm{C}, 95 \%\right.$ air, $\left.5 \% \mathrm{CO}_{2}\right)$, getting ready for the cell plating. Primary cortical neurons were extracted from E17 mouse embryos on the day of cell plating. The cell suspension was concentrated to 10 million cortical neurons per $\mathrm{mL}$ of neural culture medium. The prepared microfluidic-MEA device was first emptied, and then filled with the freshly-prepared cell suspension into the lateral chambers (Chamber 1 and 3), carefully avoiding air bubbles. The middle chamber (Chamber 2) which contains no cells was filled with the normal neural culture medium. The device and the freshly placed neurons were kept inside the incubator for long-term cell growth and neural network development. All the experimental procedures were carried out according to the Swiss federation rules for animal experiments.

(a)

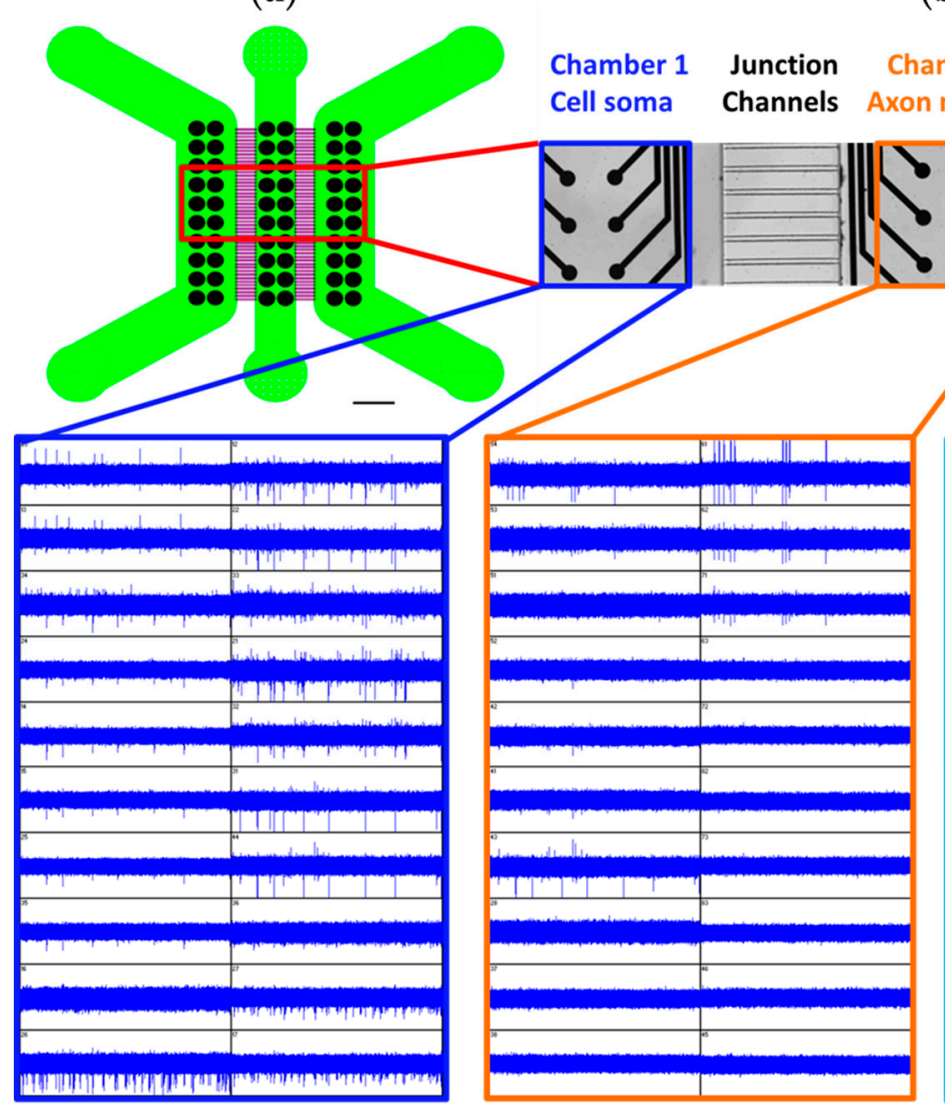

(c) (b) 
After two weeks of continuous culture in vitro, a heathy neural network was built inside the microfluidic-MEA device like in a normal culture dish. Spontaneous activities from the neural network were recorded with a MEA 1060 data acquisition system (Multi Channel Systems MCS $\mathrm{GmbH}$, Reutlingen, Germany) with 60 recording channels at 10 or $20 \mathrm{kHz}$ sampling frequency and 10 to $3000 \mathrm{~Hz}$ hardware bandpass filter (Figure 6c). Data acquisition and analysis were obtained using the MC_Rack software (Multi Channel Systems MCS GmbH). The activity signals from the neuron soma region and the axon region were recorded by the 60 electrodes (see Video S1). The bonding could not be separated afterwards in the vast majority of cases (with very rare failures occurring possibly due to the roughness or flatness of the PDMS device surface).

Comparing to other technologies such as spin-coating SU-8 onto the PDMS surface, our bonding method is simple and convenient as shown in the example of our microfluidic-MEA device, and requires only a chemical hood and an oven. The microfluidic PDMS device and MEA device can be fabricated individually and synchronously, and integrated together at the end of the process without any limitations. This flexible combination characteristic of this bonding method allows an easy fabrication of microfluidic systems and sealed chambers based on PDMS and SU-8, while avoiding leakages and allowing the obtained systems to be re-used after being cleaned. Concerning the device re-use, the device was aspirated first, then washed with DI water and treated with trypsin after use to detach the cells. In the end, the device was emptied, dried and kept in a sterile environment until the next use.

\section{Conclusions}

We present here a simple and reliable PDMS and SU-8 irreversible bonding method, which is based on the covalent bonds obtained by introducing aminosilane molecules by liquid-phase silanization. This bonding method was assessed by XPS analysis and validated by tensile strength tests. The method we propose here for irreversible bonding PDMS and SU-8 is robust on its bonding quality, flexible for integration, simple and user-friendly for manipulation, and compatible for in vitro cell culture. This makes it very useful for many applications related to PDMS and SU-8 materials, in particular microfluidics and lab-on-a-chip devices where handling of fluids needs to be performed without leakage. It was successfully applied in the fabrication of our integrated microfluidic-MEA device. This innovative integrated microdevice provides the possibility for neuroscientists to study neural electrophysiology from compartmentalized neuronal culture in vitro and investigate the communications between cell populations under different disease conditions. It will allow, more thoroughly and comprehensively, observations in neurodegenerative disease research and pharmaceutical development.

Supplementary Materials: Video S1, an example of a 10 min neural activity recording data from neuronal culture, is available online at http:/ /www.mdpi.com/2072-666X/6/12/1465/s1.

Acknowledgments: We would like to thank Prof. Jan-Anders E. Månson and and his Ph.D. student Amaël Maximilien Cohades from the Laboratoire de Technologie des Composites et Polymères (LTC) in EPFL, for their support on the tensile strength test. We acknowledge Pierre Mettraux from the Molecular and Hybrid Materials Characterization Center (MHMC) in EPFL for obtaining the XPS data. The fabrication process of MEA device was developed under the technical support from Qwane Biosciences SA, Switzerland, and we would like to thank Thibault Francfort from Qwane Biosciences SA for his help on building the manual tensile strength test set-up. We would like to thank Gabriel Safar for his advice with the manuscript. We are grateful to École Polytechnique Fédérale de Lausanne (EPFL) in Switzerland for the financial support of this research.

Author Contributions: Yufei Ren, Shun-Ho Huang conceived, designed and performed the experiments; Yufei Ren and Arnaud Bertsch analyzed the data; Marc Olivier Heuschkel contributed to the MEA fabrication; Sébastien Mosser and Patrick C. Fraering contributed to the mouse primary cortical neuron culture for testing the device; Yufei Ren and Arnaud Bertsch wrote the paper. Jia-Jin Jason Chen and Philippe Renaud co-supervised the work and reviewed the manuscript.

Conflicts of Interest: The authors declare no conflict of interest. 


\section{References}

1. Mata, A.; Fleischman, A.J.; Roy, S. Characterization of polydimethylsiloxane (PDMS) properties for biomedical micro/nanosystems. Biomed. Microdevices 2005, 7, 281-293. [CrossRef] [PubMed]

2. Fujii, T. PDMS-based microfluidic devices for biomedical applications. Microelectron. Eng. 2002, 61-62, 907-914.

3. Lorenz, H.; Despont, M.; Fahrni, N.; Brugger, J.; Vettiger, P.; Renaud, P. High-aspect-ratio, ultrathick, negative-tone near-UV photoresist and its applications for MEMS. Sens. Actuators A Phys. 1998, 64, 33-39. [CrossRef]

4. Campo, A.D.; Greiner, C. SU-8: A photoresist for high-aspect-ratio and 3D submicron lithography. J. Micromech. Microeng. 2007, 17, R81-R95. [CrossRef]

5. Abgrall, P.; Conedera, V.; Camon, H.; Gue, A.M.; Nguyen, N.T. SU-8 as a structural material for labs-on-chips and microelectromechanical systems. Electrophoresis 2007, 28, 4539-4551. [CrossRef] [PubMed]

6. Patel, J.N.; Gray, B.L.; Kaminska, B.; Wu, N.-C.; Gates, B.D. SU-8- and PDMS-based hybrid fabrication technology for combination of permanently bonded flexible and rigid features on a single device. J. Micromech. Microeng. 2013, 23, 065029. [CrossRef]

7. Patel, J.N.; Kaminska, B.; Gray, B.L.; Gates, B.D. PDMS as a sacrificial substrate for SU-8-based biomedical and microfluidic applications. J. Micromech. Microeng. 2008, 18, 095028. [CrossRef]

8. Ou, J.; Glawdel, T.; Ren, C.L.; Pawliszyn, J. Fabrication of a hybrid PDMS/SU-8/quartz microfluidic chip for enhancing UV absorption whole-channel imaging detection sensitivity and application for isoelectric focusing of proteins. Lab Chip 2009, 9, 1926-1932. [CrossRef] [PubMed]

9. Zhang, Z.; Zhao, P.; Xiao, G.; Watts, B.R.; Xu, C. Sealing SU-8 microfluidic channels using PDMS. Biomicrofluidics 2011, 5, 46503-465038. [CrossRef] [PubMed]

10. Kanagasabapathi, T.T.; Ciliberti, D.; Martinoia, S.; Wadman, W.J.; Decre, M.M. Dual-compartment neurofluidic system for electrophysiological measurements in physically segregated and functionally connected neuronal cell culture. Front. Neuroeng. 2011, 4, 13. [PubMed]

11. Zhu, M.; Lerum, M.Z.; Chen, W. How to prepare reproducible, homogeneous, and hydrolytically stable aminosilane-derived layers on silica. Langmuir 2012, 28, 416-423. [CrossRef] [PubMed]

12. Sunkara, V.; Cho, Y.K. Investigation on the mechanism of aminosilane-mediated bonding of thermoplastics and poly(dimethylsiloxane). ACS Appl. Mater. Interfaces 2012, 4, 6537-6544. [CrossRef] [PubMed]

13. Zhang, Z.; Zhao, P.; Xiao, G. The fabrication of polymer microfluidic devices using a solid-to-solid interfacial polyaddition. Polymer 2009, 50, 5358-5361. [CrossRef]

14. Martin, H.J.; Schulz, K.H.; Bumgardner, J.D.; Walters, K.B. XPS study on the use of 3-aminopropyltriethoxysilane to bond chitosan to a titanium surface. Langmuir 2007, 23, 6645-6651. [CrossRef] [PubMed]

15. Sunkara, V.; Park, D.K.; Hwang, H.; Chantiwas, R.; Soper, S.A.; Cho, Y.K. Simple room temperature bonding of thermoplastics and poly(dimethylsiloxane). Lab Chip 2011, 11, 962-965. [CrossRef] [PubMed]

16. Tang, L.; Lee, N.Y. A facile route for irreversible bonding of plastic-PDMS hybrid microdevices at room temperature. Lab Chip 2010, 10, 1274-1280. [CrossRef] [PubMed]

17. Kim, K.; Park, S.W.; Yang, S.S. The optimization of PDMS-PMMA bonding process using silane primer. BioChip J. 2010, 4, 148-154. [CrossRef]

18. Smith, E.A.; Chen, W. How to prevent the loss of surface functionality derived from aminosilanes. Langmuir 2008, 24, 12405-12409. [CrossRef] [PubMed]

19. Mollenhauer, H.H.; Morre, D.J.; Pikaard, D.; Clark, D.E. An ultrastructural evaluation of toluene toxicity using cultured mammalian cells. J. Submicrosc. Cytol. Pathol. 1990, 22, 523-527. [PubMed]

20. Ren, Y.; Kunze, A.; Renaud, P. Compartmentalized microfluidics for in vitro Alzheimer's disease studies. In Microfluidic and Compartmentalized Platforms for Neurobiological Research; Biffi, E., Ed.; Springer: New York, NY, USA, 2015; pp. 197-215.

21. Fan, R.; Yue, M.; Karnik, R.; Majumdar, A.; Yang, P. Polarity switching and transient responses in single nanotube nanofluidic transistors. Phys. Rev. Lett. 2005, 95, 086607. [CrossRef] [PubMed]

22. Ghosh, A.; Schiraldi, D.A. Improving interfacial adhesion between thermoplastic polyurethane and copper foil using amino carboxylic acids. J. Appl. Polymer Sci. 2009, 112, 1738-1744. [CrossRef] 
23. Heuschkel, M.O.; Wirth, C.; Steidl, E.-M.; Buisson, B. Development of 3-D multi-electrode arrays for use with acute tissue slices. In Advances in Network Electrophysiology; Taketani, M., Baudry, M., Eds.; Springer: New York, NY, USA, 2006; pp. 69-111.

24. Ehlers, J.-E.; Rondan, N.G.; Huynh, L.K.; Pham, H.; Marks, M.; Truong, T.N. Theoretical study on mechanisms of the epoxy-amine curing reaction. Macromolecules 2007, 40, 4370-4377. [CrossRef]

25. Nietzold, C.; Dietrich, P.M.; Ivanov-Pankov, S.; Lippitz, A.; Gross, T.; Weigel, W.; Unger, W.E.S. Functional group quantification on epoxy surfaces by chemical derivatization (CD)-XPS. Surface Interface Anal. 2014, 46, 668-672. [CrossRef]

26. Johnston, I.D.; McCluskey, D.K.; Tan, C.K.L.; Tracey, M.C. Mechanical characterization of bulk Sylgard 184 for microfluidics and microengineering. J. Micromech. Microeng. 2014, 24, 035017. [CrossRef]

(C) 2015 by the authors; licensee MDPI, Basel, Switzerland. This article is an open access article distributed under the terms and conditions of the Creative Commons by Attribution (CC-BY) license (http://creativecommons.org/licenses/by/4.0/). 